\title{
A framework for the descriptive specification of awareness support in multimodal user interfaces for collaborative activities
}

\section{Introduction}

Over the past few years, the scientific area of Ambient Intelligence (AmI) [33] has been proposing a new computing paradigm for daily life. The main aim of AmI is the intelligent adaptation of the behavior of a context-aware computational environment to our preferences and habits, so that our experiences and routines in daily life are improved [20]. AmI research builds upon advances in sensor networks, pervasive computing and artificial intelligence, and deals with work on hearing, human vision, language and knowledge, which are all related to human intelligence. Here is where AmI differs from the concept of ubiquitous computing [21]. Thus, new scenarios emerge in which new interaction devices and innovative styles of interaction are used, and where capturing information from the environment, providing notifications and serving user requests are common processes.

On the other hand, our world is collaborative. The development of complex engineering projects, scientific advances, global communications or rich social interactions are possible thanks to the joint efforts and participation of many people, for which suitable technological support is essential. Thus, groupware (software for working in groups) is omnipresent in a number of group activities ranging from working and learning to gaming and social life.

In these kinds of environments, the classic concept of interaction from the Human-Computer Interaction (HCI) field is extended, and thus a distinction between the so-called explicit interaction and implicit interaction is proposed [32]. Explicit interaction is the classical interaction that takes place between users and devices that provide services to them, while the concept of implicit interaction includes those actions carried out by users without an explicit intention, but that the system proactively captures and processes as inputs. Accordingly, user interfaces are multimodal, with more than one interaction modality operating in either parallel or not, and with transitions between explicit and implicit interactions.

Within implicit user interfaces, physiology-based systems have a great potential. These systems manage implicit models of interaction where the user's physiological signals, such as brain waves or heart rate, are monitored and transformed in real time in commands to control devices and adaptive applications [22]. In addition, an emergent line of research in $\mathrm{HCI}$ is the design of interfaces that can process sensory inputs. As Obrist et al. [27] point out, we mostly rely on sight and hearing, but it should be possible to create richer experiences for humantechnology interactions and group activities by incorporating the senses of touch, smell and even taste.

All these new concepts, settings, technologies and users' requirements entails a number of challenges that affect to collaboration support, being awareness -defined as the perception and knowledge of the activity of other members in a working group [6]- one of its most critical components, due to its potential to provide an effective collaborative experience. Moreover, up to now, most awareness mechanisms have been visual, and thus have suffered from several limitations related to the size of the screen, the drawing of the actions and the perception of changes in the display [15]. These facts suggest the use of new channels for awareness. This multimodality provides both opportunities and challenges for awareness support. In fact, recent studies suggest that in the following years, ubiquitous and ambient techniques may be much linked to awareness support in ComputerSupported Collaborative Work (CSCW) [19].

In the present age of tablets, smartphones and wearables, in this article we focus on the problems of modeling and designing the awareness support for collaborative systems based on multimodal interaction and AmI, which involves considering issues such as new devices, physiology-based functionalities and sensing technologies.

We deal with this open research problem by taking our previous works about the modeling of collaborative systems and of awareness support as a starting point [8]. Also, in some other works that we have recently carried out $[9,24,25]$ we have gone deeper in the field of collaborative systems, specifically in the field of Computer-Supported Cooperative Learning (CSCL), which we have used mainly for the case of study of teaching programming and software engineering. Awareness support has had a great importance in our works in this line. Now, our overall aim is to contribute a framework for the design of awareness support for our target collaborative systems.

This framework is based on the awareness framework proposed by Gutwin and Greenberg [14], which is one of the most commonly used when evaluating the awareness support of systems based on explicit interaction, as well as the framework of Antunes et al. [1].

The specific contribution of this work is twofold. Firstly, we propose a rich conceptual model of 
awareness support in collaborative systems based on multi-model interaction. Then, a descriptive language for specifying awareness statements is proposed to be used by groupware engineers in the stages of requirements specification and design of a collaborative system, as well as a three-step process for using these modeling tools. An awareness statement characterizes awareness actions in terms of the areas, dimensions and awareness information to which they refer to, of the inputs and outputs of the action, and of the widgets and devices involved in the underlying interactions.

The rest of the paper is organized as follows: Section 2 reviews the research areas of Context Modeling in AmI and Awareness Support in Collaborative Systems. Next, Section 3 presents our proposal of a framework for the design of the awareness support in collaborative systems based on multi-model interaction. Then, Section 4 includes a preliminary validation of the framework and examples of use to show its usefulness. Finally, Section 5 draws some conclusions and outlines some lines for future work.

\section{Related work}

\subsection{Context model in Ambient Intelligence}

Context modeling emerges as a fundamental aspect to consider when working on AmI. A wellknown definition states that context is "any information that can be characterized to define a situation of an entity", where an entity is "a person, place or object considered relevant for the interaction between a user and an application." [5].

There are several approaches of architectures and models to support context modeling. The CoBrA proposal [3] defines a centralized architecture that supports agents, places and events in an intelligent environment. It is a centralized architecture because it relies on the presence of an element called Context Broker that maintains the context model shared by the rest of the elements in the environment. This proposal includes the use of an ontology that the authors have specialized for a particular case study. Concepts in this ontology are related to the place, the broker, the location of the agent or the agent's activity.

Another interesting proposal is SoaM [36]. It proposes a distributed architecture, unlike the previous one, although it has elements in common, such as the definition of entities and agents or the use of ontologies. This proposal defines four types of information: context information, capabilities, constraints and behavior profiling.

Finally, COIVA [16] is another example of architecture for context-sensitive environments. It is focused on information visualization in intelligent environments, and tries to do context modeling by means of an ontology called PIVOn, that include views on the user, the environment, the service, the device and the visualization.

\subsection{Awareness support in Collaborative Systems}

The concept of awareness appears as a requirement to consider when developing systems that must support collaboration effectively. The idea behind awareness is to maintain users informed about who is collaborating, the actions of the other group members and even what action is expected to be the next to be executed. Examples of specific mechanisms of awareness are the use of tele-pointers, participant lists or multi-scrollbars [13]. The relationship between context and awareness is twofold: awareness services gathers knowledge from the context,,and awareness takes place within specific contexts and produces effects on them.

The main theoretical contributions in the field of awareness are the ones carried out by Gutwin and Greenberg [13, 14]. These authors define four types of awareness: informal, social, group structure and workspace, although in most of their works they focus on the last one. In particular, informal awareness refers to the overall perception of who is there and what he/she intends to do; social awareness is the information about other users that someone has in a social or conversational context; group structure awareness implies knowledge about roles, responsibilities and status of the other members; and workspace awareness provides knowledge about the interactions of the others with the workspace and its artifacts.

The workspace awareness framework these authors propose [14] is one of the few contributions in the literature that addresses the verification or checking of the awareness support in synchronous collaborative applications. In so doing, this framework proposes ten questions to be answered to determine whether a component or tool gives adequate support to workspace awareness. However, this contribution focuses on scenarios where users interact with a classical graphical user interface in which there is a shared context (set of objects that are viewed and manipulated jointly by the participants of a group 
work activity) [7]. In these explicit interaction environments, the interest is on supporting group structure and workspace awareness. In implicit interaction environments, however, the remaining two types of awareness mentioned above could become important: social awareness, because in such situations it is relevant to know how people are related to each other during the work sessions; and informal awareness, since it allows the perception of presence as is, without considering actions performed by users.

Antunes et al [1] characterize awareness as based on fundamental distinctions concerning time, place and space. They consider six kinds of spaces and five awareness types, two of which are location awareness and context awareness. Location awareness refers to the geographical and physical nature of spaces and to mobility issues; and context awareness deals with the notion of virtual spaces and navigation virtuality.

An analysis of the trends in awareness in CSCW has led to the birth of the term we-awareness [11, 34] This term refers to "the socially recursive inferences that let collaborators know that all are mutually aware of each other's awareness" [11]. Thus, the idea is that participants in a collaborative task must not only be oriented towards the same features of the environment, but they must each recursively know that the other person is so oriented [34]. Therefore, we can see how social features and shared behaviors are having a great influence in CSCW nowadays, and that it is changing the traditional foundations of awareness support, that must be reviewed and enhanced.

Also, different kinds of collaborative systems need different kinds of awareness support. An example is the CSCL systems, in which several students cooperate in order to perform a learning activity. The Shared Knowledge Awareness [4] is a specific approach in which information given to the students takes into account the knowledge acquired by the group as a whole in a collaborative learning setting.

As mentioned before, physiology-based systems emerge with new forms of interaction in the collaboration settings. A representative system is the Multimodal Music System proposed by Mealla et al. [22], a prototype of multimodal system for collaborative sound generation and control, combining physiological computing and a tabletop interface. The system uses sensors for capturing ECG (electrocardiography) and EEG (electroencephalography) signals. Their authors state that, in the creation and control of sounds, brain and body signals may be powerful indicators of performer's emotional and cognitive states during collaboration, facilitating music anticipation and interpersonal synchronization.

\subsection{Notations and frameworks for collaborative work and awareness modeling}

Two well-known task-based notations for specifying multi-user systems are CTT [28, 29] and CIAN [23]. They lack support to describe multimodal aspects. The COMM notation [17] extends the CTT notation by introducing two concepts, the interactive role and the modal task, and considering five relationships as temporal operators applied to concrete tasks, in order to look at the specification of multiuser multimodal user interfaces.

$\mathrm{Ju}$, Lee \& Klemmer [18] presented a framework for implicit interaction that divides the space of possible interactions along the two axes of attentional demand and initiative. Attentional demand refers to the degree of cognitive and perceptual load imposed on the user by the system, and ranges from background to foreground interactions. While initiative is an indicator of how much presumption the systems uses in the interaction, ranging from reactive, where interactions are initiated and driven by the users, to proactive interactions, where interactions are inferred and initiated by the system.

Rittenbruch [31] proposed the Atmosphere framework for the realization of contextual awareness, trying to avoid the lack of context in collaboration asynchronous settings by providing mechanisms to model and represent the context in combination with awareness services. The framework is based on two concepts: spheres, as structural representations of contexts within the system; and contextors, as active methods expressed as activities and actions.

Teruel et al [35] have developed the CSRMF framework for the specification of requirements in collaborative systems. It consists of a modeling language, a set of guidelines for the design of the systems and a tool that supports the framework's use. In CSRMF, awareness support has a great importance, and thus an awareness resource, which is related to the tasks and roles present in the system being specified, is one of its components. This work is also inspired in the concepts of workspace awareness presented in [14].

\subsection{Discussion}

In this work, we approach the problems of awareness design in systems supporting collaborative ac- 
tivities in rich and complex scenarios and contexts. In such settings, not only the shared context but also the shared environment (which additionally includes the physical context in which the interaction takes place) needs to be considered.

The awareness frameworks and works presented above fails at fulfilling the problems identified since they do not approach the shared environment concept and they are not specifically oriented to AmI. However, we think that an additive combination of workspace, social, informal and group-structure awareness together with the conceptualizations for location and context awareness is a promising approach.

On the other hand, the set of awareness conceptualizations reviewed do not propose descriptive mechanisms for awareness support. In addition, task-based notations for interactive systems, which could be applicable here, do not include primitives for modeling awareness.

Finally, we inspire in the ontologies and systems for context modeling and in the Atmosphere framework [31] in order to identify significant concepts useful to characterize the awareness statements and the underlying interaction, such as event, action, device and place.

\section{Scenarios of application}

In this section, we explain in which kind of situations will be useful our approach (presented in the next section). Firstly, it is worth noting that we deal with systems that support collaborative activities. In such activities, many users work together in order to achieve any kind of goal. The collaboration may be of a synchronous nature (if users collaborate at the same time) or of an asynchronous one (if not all users are interacting at the same time). Collaborative systems usually imply the existence of a shared environment, which is made up of the objects shared by the different users. This shared environment can be implemented in different shared spaces, which may be spaces of real life in ubiquitous or pervasive situations.

In general, the systems we support are of a multimodal nature. That is, many different kinds of inputs and outputs have to be handled. This supposes a problem when designing such systems, as it is often difficult to have clear how to integrate data from input devices and how to generate relevant outputs taking advantage of all output devices. Input data can be obtained from more traditional devices (keyboard, mouse, etc.) or from more novel ones (gesture, speech, etc.). Even, sometimes input data is get from sensors that the user is not aware of. In these cases, we will talk about implicit interaction, as there is not an explicit action carried out by the user.

As characterized, these systems can find an application in several fields. For example, in leisure, the support given by an amusement park can fit in this description, as many users can collaborate in their visit to the park and they may perform actions in an implicit way when going through the different attractions and shows in the park. Later, in Section 5 we will mention some specific examples.

\section{A framework for the descriptive specification of awareness support in multimodal collaborative user interfaces}

In a previous work [8], we developed a framework in which we specified the inputs and outputs we understand that have to be taken into account when considering awareness issues in each dimension considered by Gutwin and Greenberg in its awareness framework. These inputs and outputs were specified for both explicit interaction and implicit interaction situations.

In this work, we extend the awareness dimensions considered in [8] and thus we present a framework for specifying awareness support in situations in which multimodal interfaces are present and collaborative activities are carried out, as outlined above.

The framework includes the following elements (Figure 1):

Conceptual framework. It includes two views: on the one hand, we have the awareness conceptualization, based on three components: awareness areas, awareness dimensions, and information elements; on the other hand, we have developed a catalog of widgets and devices for awareness support, where specific elements for giving awareness support are identified.

- Descriptive specification. It consists of a way of specifying, in a pseudo formal way, the event and context that may lead to any awareness notification and the output in terms of awareness support linked to such information.

- Process of application. It is made up by a series of steps that may be followed when willing to apply this framework to a real situation in which one groupware system with the aforementioned features should be provided with awareness support. 


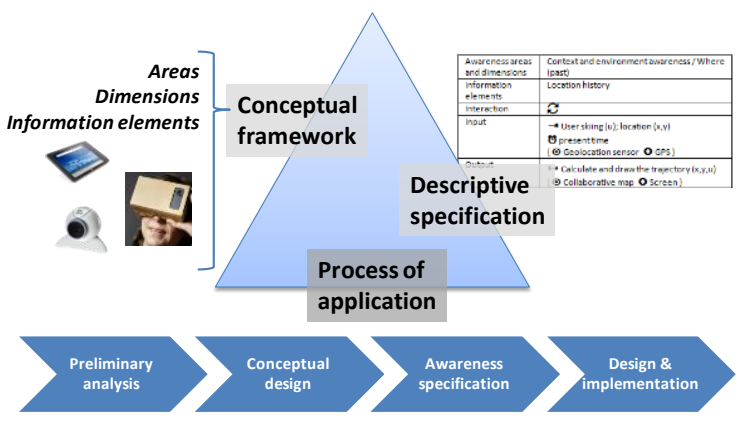

Figure 1. Structure of the framework.

In the following subsections, we describe each one of the three elements in the framework, so that we make clear how it is organized and how it can be applied.

\subsection{Conceptual framework}

The first element in the framework is the conceptual framework, which is the basis for the rest of the framework. Specifically, two tables are going to be shown, one referred to information elements for each dimension of awareness and another one for widgets and devices for awareness support.

In the first table, we are going to detail the dimensions and information elements for which awareness support is going to be defined. The table is based on relevant works about awareness support [1, 12, 14] and also in our previous experience on the field [8].

The dimensions and information elements we are going to handle are based on the ones that are present in the work by Gutwin and Greenberg [14] about workspace awareness (see Table 1). In the case of context and environment awareness, we have reinterpreted their conceptualization for this new kind of approach, reusing the same elements considered for workspace awareness. The idea is that we try to exceed in this area the concept of workspace, keeping the same dimensions and elements in the scope of environment and context, which is a wider concept. Workspace awareness is oriented towards the senses of sight, mainly, and hearing. With this extension we are trying to cover some other senses for which more innovative devices and widgets begin to exist. The where element (both for present and past actions) deals with spatial and virtual location and the information about mobility referred as location in [1], whereas the remaining elements reflect the virtual world or space and the navigation within the context.
The only element we have added is the one about senses, which we have categorized in a new What \& Where dimension within the awareness area of environment and context. This element is relevant in information collected by senses such as smell. In such cases, information felt is relevant (What), but also the location in which that information has been felt (Where).

Together with context and environment awareness and workspace awareness, some other types of awareness should be considered, as many authors note $[12,14]$. Specifically, we have included in our framework the following: informal awareness, social awareness and group-structural awareness. In consequence, we have five types of awareness, taking into account that sometimes they will overlap and other times they will be interdependent.

This conceptualization of awareness areas, dimensions and elements is going to be useful for us as we are going to detail later all the mechanism for awareness support according to these elements. In Table 1, we show the dimensions that are included for each awareness area and the information elements that are considered for each dimension.

The second component of the conceptual framework is a catalog of widgets and interaction devices for awareness support, shown in Table 2. For our purposes, and according to [30], widgets are small embeddable applications that can usually be executed within runtime containers. Users' interaction with groupware systems, both implicit and explicit, takes place through devices and widgets, interfaces techniques or controllers. We have put together all these elements on this list so that it is clear which means are available for both collection information and providing it to users when supporting awareness. We have made up the table starting from works such as $[2,10,14]$, some of which are previous works in which we explored these concepts. 


\begin{tabular}{|c|c|}
\hline Dimension & Information element \\
\hline \multicolumn{2}{|c|}{ Awareness area: Workspace awareness (WA) } \\
\hline Who & $\begin{array}{l}\text {-Presence } \\
\text {-Identity } \\
\text {-Authorship }\end{array}$ \\
\hline What & $\begin{array}{l}\text {-Action } \\
\text {-Intention } \\
\text {-Artifact }\end{array}$ \\
\hline Where & $\begin{array}{l}\text {-Location } \\
\text {-Gaze } \\
\text {-View } \\
\text {-Reach }\end{array}$ \\
\hline How & $\begin{array}{l}\text {-Action history } \\
\text {-Artifact history }\end{array}$ \\
\hline When & -Event history \\
\hline Who (past) & -Present history \\
\hline Where (past) & -Location history \\
\hline What & -Action history \\
\hline \multicolumn{2}{|c|}{$\begin{array}{l}\text { Awareness area: Context and environment awareness } \\
\text { (CEA) }\end{array}$} \\
\hline Who & $\begin{array}{l}\text {-Presence } \\
\text {-Identity } \\
\text {-Authorship }\end{array}$ \\
\hline What & $\begin{array}{l}\text {-Action } \\
\text {-Intention } \\
\text {-Artifact }\end{array}$ \\
\hline Where & $\begin{array}{l}\text {-Location } \\
\text {-Gaze } \\
\text {-View } \\
\text {-Reach }\end{array}$ \\
\hline What \& Where & -Senses \\
\hline How & $\begin{array}{l}\text {-Action history } \\
\text {-Artifact history }\end{array}$ \\
\hline When & -Event history \\
\hline Who (past) & -Present history \\
\hline Where (past) & -Location history \\
\hline What & -Action history \\
\hline \multicolumn{2}{|c|}{ Awareness area: Informal awareness (IA) } \\
\hline Informal information & $\begin{array}{l}\text {-Informal knowledge and casual } \\
\text { interaction }\end{array}$ \\
\hline \multicolumn{2}{|c|}{ Awareness area: Social awareness (SA) } \\
\hline Social information & $\begin{array}{l}\text {-Emotional state } \\
\text {-Other nonverbal cues }\end{array}$ \\
\hline \multicolumn{2}{|c|}{ Awareness area: Group-structural awareness (GSA) } \\
\hline Group structure & $\begin{array}{l}\text {-Roles and responsibilities } \\
\text {-Group structure }\end{array}$ \\
\hline
\end{tabular}

Table 1. Areas, dimensions and information elements in our awareness framework.

\begin{tabular}{|c|c|}
\hline Widgets, controllers or tools & Devices (Input/Output) \\
\hline \multicolumn{2}{|c|}{ Sense or category: Sight } \\
\hline $\begin{array}{l}\text { Participant list } \\
\text { Radar view } \\
\text { Fish-eye view } \\
\text { Tele-pointer } \\
\text { Coordination semaphores } \\
\text { Group progress bar } \\
\text { Group scrollbar } \\
\text { Event list } \\
\text { Video conferencing } \\
\text { Collaborative editor } \\
\text { Collaborative map } \\
\text { Meeting agenda } \\
\text { Physiological properties panel } \\
\text { User emotional representation }\end{array}$ & $\begin{array}{l}\text { Screen }(0) \\
\text { Smart glasses }(0) \\
\text { Virtual reality glasses } \\
(0) \\
\text { Camera (I) }\end{array}$ \\
\hline \multicolumn{2}{|c|}{ Sense or category: Touch } \\
\hline- & $\begin{array}{l}\text { Mouse (I) } \\
\text { Keyboard (I) } \\
\text { Data glove (I) } \\
\text { Fingerprint sensor (I) } \\
\text { Pressure bracelet (O) }\end{array}$ \\
\hline \multicolumn{2}{|c|}{ Sense or category: Sight-Touch } \\
\hline- & $\begin{array}{l}\text { Touch screen }(\mathrm{I} / \mathrm{O}) \\
\text { Interactive table }(\mathrm{I} / \mathrm{O}) \\
\text { Interactive whiteboard } \\
(\mathrm{I} / \mathrm{O}) \\
\text { Smart watch }(\mathrm{I} / \mathrm{O})\end{array}$ \\
\hline \multicolumn{2}{|c|}{ Sense or category: Hearing } \\
\hline $\begin{array}{l}\text { Speech recognition } \\
\text { Voice synthesizer } \\
\text { Audio cues } \\
\text { Proximity sonar } \\
\text { Sound and music generators } \\
\text { Sound and music processors }\end{array}$ & $\begin{array}{l}\text { Speaker (O) } \\
\text { Earphones (O) } \\
\text { Microphone (I) }\end{array}$ \\
\hline \multicolumn{2}{|c|}{ Sense or category: Smell } \\
\hline Nose controller & $\begin{array}{l}\text { Electronic nose }(\mathrm{I}) \\
\text { Smell generator }(\mathrm{O})\end{array}$ \\
\hline \multicolumn{2}{|c|}{ Sense or category: Bodily properties } \\
\hline $\begin{array}{l}\text { Emotion recognition controller } \\
\text { Facial expression generator }\end{array}$ & $\begin{array}{l}\text { Eye tracker }(\mathrm{I}) \\
\text { Activity tracker (I) } \\
\text { Heart ECG }(\mathrm{I}) \\
\text { Brain EEG }(\mathrm{I}) \\
\text { Heart rate bracelet }(\mathrm{I}) \\
\text { Thermometer }(\mathrm{I}) \\
\end{array}$ \\
\hline \multicolumn{2}{|c|}{ Sense or category: Environment } \\
\hline $\begin{array}{l}\text { Geolocation sensor } \\
\text { Shared digital clock }\end{array}$ & $\begin{array}{l}\text { GPS (I) } \\
\text { Gyroscope (I) } \\
\text { Accelerometer (I) } \\
\text { Speedometer (I) } \\
\text { RFID receiver (I) } \\
\text { RFID activator (O) }\end{array}$ \\
\hline
\end{tabular}

Table 2. Widgets, controllers, tools and devices to use in the framework (the list is not exhaustive). 
The information in the table is grouped by senses and similar categories: sight, touch, sight together with touch, hearing, smell, bodily properties and environment. For each category, we have first listed those widgets or controllers used to handle the information related with it. And then, in the second column, we include which devices can be used for both collecting information (input, I) or providing with it (output, O). It can be seen how we are dealing with information of a multimodal nature, collected in many ways and notified also by means of quite different means.

\subsection{Descriptive specification for awareness actions}

Once the conceptual framework of the proposal has been defined, it is now the moment to introduce the specification we propose to define requirements in a system that deals with multimodal situations of implicit interaction. Our idea when defining this specification was to have an easy way to define which inputs and outputs related with awareness had to be taken into account. This way, requirements related to awareness may be easily collected and the design of the suitable awareness support could be easily carried out.

The specification consists of the definition of an awareness card (see Table 3) which includes the data needed for a given information element of the ones that appeared in the conceptual framework. Tasks in a collaborative system may require more than one awareness action.

At the time of building the notation, we took into account some principles stated by Moody [26] for visual notations, such as Semiotic Clarity (1:1 correspondence between constructs and symbols), Perceptual Discriminability (different symbols are distinguishable), Semantic Transparency (the appearance suggests the meaning -partially-), Complexity Management (boxes for dealing with complexity), Dual Coding (text to complement graphics), and Graphic Economy (a number of symbols cognitively manageable).

Specifically, the data that should be provided for each information element is the following (see Table 3):

- Awareness area and dimensions. Here, the user of the framework will specify the awareness area and the dimensions in which the information elements are framed, as specified in the conceptual framework. In the case of situations of explicit interaction, this area will usually be workspace awareness, whereas in the case of situations of implicit interaction, context and environment awareness will be the most usual area to be specified.

- Information elements. The name of the specific information element or elements to be handled is specified here.

- Interaction. Prior to specifying the data about the interaction, it is needed to detail whether the interaction is of an explicit (classic) or implicit nature.

- Input of the interaction. Here, the data about how the input of information is treated is detailed. These are the items that have to be specified:

- Event and information. At this point, the specific event to be intercepted as a relevant input for awareness intervention and the information about it are detailed. They are marked with an arrow.

- Time. The time in which the interaction takes place is specified here. It is marked with a clock.

- Source user interface widgets, controllers or tools. Here, the widgets, controllers or tools used to catch the information are specified. These elements are usually chosen from the ones specified in the conceptual framework. This data is marked with a circle with a point inside it.

- $\quad$ Source devices. Source devices are the physical devices used to actually obtain the information about the interaction that has taken place. They may be some of the included in the conceptual framework. They are marked with an empty circle.

- Output of the interaction. In the same way that is carried out about the inputs, some items about the output of the interaction have also to be specified. They are the following:

- Actions and parameters. They are the actions that should occur in order to notify users or groups in some way as a consequence of some inputs, and the parameters, which make up the content needed for the actions to take place. They are marked with double arrows.

- Target widgets and containers. They are the elements in the user interface that allow that the notification specified in the action element takes place. The widgets and containers are usually some from the list of the conceptual framework. They are marked with a circle with a point inside it.

- Target devices. They are the physical devices that support the notification. They may be 
some of the specified in the conceptual framework. They are marked with an empty circle.

- Description. The last data that is collected in a specification is an informal description in which any piece of information that does not fit in the remaining categories can be explained.

It can be seen how the data that are specified about inputs and outputs of information handled in situations of implicit interaction is similar that in the case of explicit interaction. As it has been already stated, one of the contributions of this work is how we handle this information in a way that is comparable to how explicit interaction is treated. Obviously, implicit interaction has its own distinctive features, due to factors such as its strong multimodal nature. The widgets and devices used for implicit interaction are quite different from those used for explicit interaction The latter will be classic widgets and devices such as the mouse, the keyboard, etc., whereas the former will be more innovative widgets, such as geolocation sensors, bracelets, accelerometers, etc.

With all those elements, awareness information and support can be specified in a descriptive way. This may be of great help when analyzing the requirements of an interactive application, mainly if we are dealing with an application of a multimodal nature in which many different inputs and outputs from different sources and targets are being handled. In table 3 it is depicted all the aforementioned components of an awareness card.

\begin{tabular}{|l|l|}
\hline $\begin{array}{l}\text { Awareness } \\
\text { areas and } \\
\text { dimensions }\end{array}$ & $\begin{array}{l}\text { Awareness areas and dimensions af- } \\
\text { fected }\end{array}$ \\
\hline $\begin{array}{l}\text { Information } \\
\text { elements }\end{array}$ & $\begin{array}{l}\text { Information elements from areas and } \\
\text { dimensions }\end{array}$ \\
\hline Interaction & $\begin{array}{l}\text { Modality of interaction: } \\
\text { Explicit A In | Implicit }\end{array}$ \\
\hline Input & $\begin{array}{l}\rightarrow \text { event \& information } \\
\text { @ time of the interaction } \\
\{\odot \text { source user interface widgets, con- } \\
\text { trollers or tools } \\
\text { O source devices }\}\end{array}$ \\
\hline Output & $\begin{array}{l}\mapsto \text { actions \& parameters } \\
\{\odot \text { target widgets \& containers } \\
\text { O target devices }\}\end{array}$ \\
\hline Description & $\begin{array}{l}\text { Description of the interaction and of the } \\
\text { awareness to provide }\end{array}$ \\
\hline
\end{tabular}

Table 3. Structure of an awareness card.

\subsection{Process for the application of the framework}

It is now the moment to define how we are going to use the awareness conceptualization and the awareness specification to facilitate the development of interactive applications. Specifically, we are going to detail some steps for the application of the framework that may be carried out during the phase of requirements analysis in a typical software development process. It will be also interesting to frame the use of the framework in a User-centered design process, which may be of the MBUID kind or similar. The specific steps are the following:

1.- Preliminary analysis. The first thing that has to be clear in order to specify the awareness support of the application is the main goals of the application and the interactive tasks that it will have to support. At this step, usual techniques for requirements analysis (e.g., Use cases) and collaborative task modeling (e.g., CTT) will be used. The result of this step will be those specifications of goals and tasks that will help developers in the following steps.

2.- Conceptual design. Once the general requirements about the application has been collected, the information elements for which awareness support is needed have to be identified. Here, the nature of the interaction (explicit or implicit) must be also decided. The outcome of this step should be a description of the collaborative support and of the architecture of the awareness support on the basis of the models proposed in Section 4.1.

3.- Awareness specification. This is the step in which the particular data about the explicit or implicit interaction is defined. Both input and output information has to be specified following the awareness descriptive specification technique shown in Section 4.2 , i.e., the awareness cards. In this step, the engineer deals with widgets and devices. If additional remarks have to be included, the description field may be used as well.

4.- Design and implementation of the awareness support. Once the descriptive specification has been developed, the requirements analysis related with awareness support has finished. Therefore, now this specification may be used in the design step of the development process being followed. The idea is that the specifications will be of great help for this step, as some design decisions will be immediate to take. For instance, some widgets to add to the user interface and some content that may be handled are already decided and can be directly included to the design and implementation of the groupware system. 
In this paper, we are covering phases 2 and 3 of the aforementioned process. Phases 1 and 4, which are the link between our proposal and a typical software development process, may be explained in detail and validated in further papers.

\section{Validation study}

\subsection{Description and method}

As a preliminary validation of the framework, we have carried out a conceptual and empirical validation. In order to conduct the study we have considered the following research questions:

- RQ1: Validity - Are the framework, its models and its description language useful and valid in order to describe awareness support?

- RQ2: Ease of use - Is the framework easy to use when describing awareness support, as well as understandable?

- RQ3: Fruitfulness - When used, does the framework generate results that are useful for users?

Below, we present the activities to test the research questions based on case studies, awareness specification tasks in a problem-solving approach, questionnaires for subjective evaluation, and evaluation of solutions (awareness cards). Later, in Section 5.4, we discuss the results.

\subsection{Examples of awareness description}

As a first validation, we have tested the framework -its structural components, the catalogue of widgets and devices, and the description language- by using it to model real (or potentially real) awareness requirements in the user interfaces of three systems of different nature.

\section{Case study \#1: Tele-pointers}

The first case study (CS1) is a common functionality consisting in several tele-pointers in a collaborative modeling tool (or tele-cursors in a collaborative editor). This type of awareness falls in the dimension where within the area of workspace awareness (Table 4), and the information element that is related is location. The input for providing the awareness is any movement of the user's pointer using his/her mouse or keyboard. The output is the representation of the tele-pointer of the user with the corresponding color in the modeling tool of all the users and, according to the card description, updating the user' area of interest in the radar view. Finally, the interaction modality is explicit interaction.

\begin{tabular}{|c|c|}
\hline $\begin{array}{l}\text { Awareness areas } \\
\text { and dimensions }\end{array}$ & Workspace awareness / Where \\
\hline $\begin{array}{l}\text { Information } \\
\text { elements }\end{array}$ & Location \\
\hline Interaction & Aling \\
\hline Input & $\begin{array}{l}\rightarrow \text { Mouse movement event; user(u,color); } \\
\text { position }(x, y) \\
\text { ৫) present time } \\
\{\bigcirc \text { Collaborative editor } \mathbf{O} \\
\text { Mouse|Keyboard }\}\end{array}$ \\
\hline Output & $\begin{array}{l}\mapsto \text { Update: tele-pointer(x,y,u,color }), \text { radar } \\
\text { view area(u) } \\
\{\odot \text { Tele-pointer; Collaborative editor; Radar } \\
\text { view } \\
\text { O Screen }\}\end{array}$ \\
\hline
\end{tabular}

Table 4. The awareness card for case study \#1.

\section{Case study \#2: Amusement park}

The second case study (CS2) refers to an amusement park, where a group of visitors enjoy the different services provided, such as attractions, shows or restaurants. This is based on a real implementation within our research projects. The visit is usually carried out following a roadmap previously set up or discussed on the fly. In such settings, the members of the group should have knowledge about aspects such as where the remaining team members are or whether a show they are willing to attend is about to begin.

In this case, interaction is implicit, since the user is not aware of being interacting with the system neither providing it with data (Table 5). The awareness statement is characterized as belonging to the context and environment awareness area and when dimension. Since the time is the trigger variable that fires the awareness statement, the information element selected is event history. When a user arrives at an attraction or show approximately at the time programmed, a number of awareness actions are carried out: the update of the localization of the user in the map, the update of the meeting agenda and the group state, and also the playing of an audio cue. 


\begin{tabular}{|c|c|}
\hline $\begin{array}{l}\text { Awareness areas } \\
\text { and dimensions }\end{array}$ & $\begin{array}{l}\text { Context and environment awareness / } \\
\text { When }\end{array}$ \\
\hline $\begin{array}{l}\text { Information } \\
\text { elements }\end{array}$ & Event history \\
\hline Interaction & $\mathcal{X}$ \\
\hline Input & $\begin{array}{l}\rightarrow \text { Arrival to a location }(x, y) ; \text { user }(\mathrm{u}) ; \\
\text { appointment in the meeting agenda(a) } \\
\text { (ิ) present time } \\
\{\odot \text { Geolocation sensor } 0 \text { GPS }\}\end{array}$ \\
\hline Output & $\begin{array}{l}\mapsto \text { Update and display: location }(x, y, u), \\
\text { meeting agenda }(a, u), \text { state }(u) ; \\
\text { play an audio cue } \\
\{\odot \text { Audio cue } 0 \text { Speaker }\} \\
\{\text { ○ List of participants; Group state; } \\
\text { Meeting agenda } \\
\text { O Screen }\}\end{array}$ \\
\hline
\end{tabular}

Table 5. The awareness card for case study \#2.

\section{Case study \#3: Skiing in group}

The last case study (CS3) is based on a fictitious system. Let us suppose a smartphone app for skiing with colleagues. The downhill trajectory of each participant is shown in a map and the proximity among skiers is notified by means of both a sonarlike audio cue and a vibrating bracelet in the arm, in order to avoid collisions among them. Skiers can bring earphones to better hear the cues.

In this system, two awareness cards are needed to describe two functions: drawing the trajectory and checking the proximity. In both cases, the interaction is implicit and the awareness area is the context and environment awareness. In the first card (Table 6), the where (past) dimension and the location history information element define the awareness support, which consists in registering the change of location using the geolocation sensor from the GPS, and then drawing the trajectory in the map.

\begin{tabular}{|c|c|}
\hline $\begin{array}{l}\text { Awareness areas } \\
\text { and dimensions }\end{array}$ & $\begin{array}{l}\text { Context and environment awareness / Where } \\
\text { (past) }\end{array}$ \\
\hline $\begin{array}{l}\text { Information } \\
\text { elements }\end{array}$ & Location history \\
\hline Interaction & $\widetilde{X}$ \\
\hline Input & $\begin{array}{l}\rightarrow \text { User skiing }(\mathrm{u}) \text {; location }(\mathrm{x}, \mathrm{y}) \\
\text { ↔ present time } \\
\{\odot \text { Geolocation sensor } 0 \text { GPS }\}\end{array}$ \\
\hline Output & $\begin{array}{l}\mapsto \text { Calculate and draw the trajectory }(\mathrm{x}, \mathrm{y}, \mathrm{u}) \\
\{\odot \text { Collaborative map } \bigcirc \text { Screen }\}\end{array}$ \\
\hline
\end{tabular}

Table 6. First awareness card for case study \#3.

The action corresponding to the second card (Table 7) refers to the notification of the proximity in two ways: with the proximity sonar and with the vibrating bracelet. In this case, a conditional sentence is used to query for the proximity threshold.

\begin{tabular}{|c|c|}
\hline $\begin{array}{l}\text { Awareness areas } \\
\text { and dimensions }\end{array}$ & Context and environment awareness / Where \\
\hline $\begin{array}{l}\text { Information } \\
\text { elements }\end{array}$ & Location \\
\hline Interaction & $\mathcal{X}$ \\
\hline Input & $\begin{array}{l}\rightarrow \text { User skiing(u); location }(x, y) \text {; group } \\
\text { members }(g) \\
\text { ↔ present time } \\
\{\odot \text { Geolocation sensor } \mathbf{O} \text { GPS }\}\end{array}$ \\
\hline Output & $\begin{array}{l}\mapsto \text { Calculate distances }(u, g, x, y, \text { distance); } \\
\text { if distance<threshold then activate audio } \\
\text { cue }(u) \text { and vibration }(u) \\
\{\bigcirc \text { Proximity sonar } \boldsymbol{O} \text { Speakers } \mid \text { Earphones }\} \\
\{\bigcirc \text { Vibrating bracelet controller } \boldsymbol{O} \text { Vibrating } \\
\text { bracelet }\}\end{array}$ \\
\hline
\end{tabular}

Table 7. Second awareness card for case study \#3.

\subsection{External assessment}

In order to carry out an additional assessment, we prepared a study in which some students, playing the role of requirements engineers and evaluators, took part. These students $(\mathrm{N}=16)$ were enrolled in the third year of Computer Science studies (BSc) at the University of Castilla - La Mancha (Spain), and, among others, they were studying the subject of Human-Computer Interaction (second semester). All the students participating had a similar level of knowledge and skills.

Participants were given a 40 minutes lesson about the topic of Collaborative Systems and Awareness, and then an expert in the framework presented them its components during 20 minutes. Next, they were requested to review the solutions to the three problems of awareness description presented in Section 5.2, and to solve three other problems slightly more complex (CS4, CS5 y CS6), for which they were be able to spend 45 minutes. Finally, they filled out a questionnaire as explained below.

\section{Case study \#4: Social running}

Case study \#4 (CS4) is partially inspired in the Racefully system ${ }^{1}$. This is a social system for running with people from all around the world, supporting inviting people and starting a distributed running. When running, the system shows in a graphical way the distance run and the total running time, and a

\footnotetext{
${ }^{1}$ http://raceful.ly/
} 
built-in commentator gives regular audio updates about the pace and the position of the runner with respect to the other runners.

Case study \#5: Music performance in an interactive tabletop

Multimodal music composition is the domain of case study \#5 (CS5), inspired in the Reactable system $^{2}$. This consists in an interactive tabletop designed to provide interaction with sound through objects on a multi-touch enabled screen. Different modules are available for sound generation, effect processing, sequencing and control. Players spread out around the tabletop and can manipulate the musical objects. One of them participates providing two physiological signals, the heart rate and brain electroencephalography, in order to generate and control the sound.

\section{Case study \#6: Virtual nature excursion}

Finally, case study \#6 (CS 6) is based on a fictitious setting in which we have a guide in a nature space and two people with disabilities, called observers, in their homes. Specific equipment (including electronic nose, vibrating bracelet, smell generator, smart glasses, etc.) is available so that the groupware they use can support three main functions:

- the distribution of the smell of the nature space to the observers;

- the observers' command of the direction to follow by the guide in his/her exploration, through two vibrating bracelets;

- and the representation of the emotions felt by the observers to the guide in his/her smart glasses.

\subsubsection{Participants' opinion through questionnaires}

As stated before, students were invited to fill in a questionnaire once reviewed the solutions provided to problems CS1, CS2 and CS3, and approached problems CS4, CS5 and CS6. The questionnaire used Likert scales and contained three sections:

- Section \#1 was about the participants' opinion about the framework in terms of correctness, complexity and usefulness.

- In Section \#2 we asked the participants about the understandability of each awareness area.

\footnotetext{
2 http://reactable.com/
}

- Lastly, in Section \#3 participants chose the three elements in the framework that they understood to be more useful.

Therefore, in the first section of the questionnaire participants expressed their opinion about the awareness descriptions (awareness cards) of CS1, CS2 and CS3 (see Section 5.2) in terms of three concepts:

- Correctness: the degree in which the specific awareness descriptions correctly expressed the awareness requirements formulated.

- Complexity: the degree of difficulty of understanding the specific awareness descriptions.

- Usefulness: the degree in which the framework and its components are, in general, useful to express awareness requirements.

To quantify each indicator, participants answered a single question with a six-point Likert scale ranging from 0 (very low degree) to 5 (very high degree). Table 8 shows the average values and standard deviation of each variable.

\begin{tabular}{|l|c|c|}
\hline Indicators & M & SD \\
\hline Correctness & 4.08 & 0.27 \\
\hline Complexity & 2.23 & 1.25 \\
\hline $\begin{array}{l}\text { Usefulness of the framework (areas, } \\
\text { dimensions and information elements) }\end{array}$ & 3.92 & 0.73 \\
\hline $\begin{array}{l}\text { Usefulness of the catalogue of widgets and } \\
\text { devices }\end{array}$ & 4.31 & 0.72 \\
\hline $\begin{array}{l}\text { Usefulness of the descriptive awareness } \\
\text { language (awareness cards) }\end{array}$ & 3.92 & 0.73 \\
\hline
\end{tabular}

Table 8. Correctness, complexity and usefulness indicators (M: mean; SD: standard deviation).

The three usefulness indicators obtained a valuation around 4 , being the catalogue of widgets and devices the more usefulness indicator in opinion of the evaluators. Similarly, they found the specific awareness descriptions quite correct, and the degree of complexity was near 2 , which means that the complexity of the solutions is not significant.

To deepen in the property of complexity, we asked the evaluators for the general understandability of the framework as a structure for modeling awareness in the different areas. Participants' opinion was collected in the same way than before, that is, with one question for each awareness area that was given a value from 0 to 5 . The results are shown in Table 9 . The workspace and group-structural awareness areas 
were the most understandable, while the informal and social were the least understandable.

\begin{tabular}{|l|r|r|}
\hline Awareness area & M & SD \\
\hline Workspace awareness (WA) & 3.56 & 1.15 \\
\hline $\begin{array}{l}\text { Context and environment awareness } \\
\text { (CEA) }\end{array}$ & 3.19 & 1.28 \\
\hline Informal awareness (IA) & 2.56 & 0.96 \\
\hline Social awareness (SA) & 2.56 & 1.15 \\
\hline Group-structural awareness (GA) & 3.56 & 1.31 \\
\hline
\end{tabular}

Table 9. Understandability of the framework's awareness areas (M: mean; SD: standard deviation).

An interesting question to analyze (section three in the questionnaire) is which are the most and least useful components in the framework. Thus, with respect to awareness areas, the information elements of Presence, Authorship and Action were evaluated as the three most significantly useful, while the Gaze, View and Location history were seen as the least useful, all them from the Workspace Awareness area.

In relation to widgets, the Participant list from the Sight category, the Speech recognition from the Hearing category, and the Geolocation sensor from the Environment category were the most useful, and the User emotional representation from the Sight category and the Emotion recognizer from the Bodily properties category were the least useful.

Finally, regarding devices, the ones related with Smell were seen as of little usefulness, whereas the identified as most useful where the Screen from the Sight category and the Mouse from the Touch category.

\subsubsection{Participants' solutions to the problems outlined}

The students, working as engineers, built the awareness cards corresponding to the three problems proposed (CS4, CS5 and CS6), specifying this way the awareness requirements expressed for the problem formulations. A total number of 64 awareness cards were evaluated using again a sixpoint Likert scale by an expert teacher in the HCI and CSCW (Computer-supported Cooperative Work) subjects. The variables evaluated are shown in Table 10. The solutions to the problems carried out for a same student were evaluated as a whole, so that the table contains the average value of all the students. The evaluation points were assigned at the level of component of the solution, that is to say, of the awareness cards. Since these three problems are relatively complex, and due to the little time available for the students to learn the description techniques and for the problem solving, in our opinion the results are not too much significant but for comparing the use of the different components and for drawing general conclusions.

For instance, it is interesting to highlight that the students failed at identifying correctly the awareness dimensions and information elements, while they identified much better the awareness area. Specifically, the selection of the correct awareness information element was the worst skill as suggested by the work carried out in the awareness cards. Among the moderately good results, the students modeled correctly the modality of interaction, the specification of inputs (events) and outputs (actions), and the devices involved in the interaction. On the contrary, the selection of suitable awareness widgets is considered a poor result.

\begin{tabular}{|l|r|r|}
\hline Solution component & M & SD \\
\hline Awareness area & 3.69 & 1.14 \\
\hline Awareness dimension & 2.37 & 1.31 \\
\hline Awareness information element & 1.83 & 1.04 \\
\hline Modality of interaction & 3.60 & 0.86 \\
\hline Events & 3.37 & 1.13 \\
\hline Actions & 3.42 & 1.41 \\
\hline Widgets & 2.02 & 1.24 \\
\hline Devices & 3.45 & 1.26 \\
\hline
\end{tabular}

Table 10. Evaluation of the students' solution to CS4, CS5 and CS6.

\subsubsection{Correlation study}

Finally, we have analyzed the degree of association between all the indicators considered in this evaluation (participants' opinion and solutions). For this purpose, non-parametric methods for the study of the correlation were used (Spearman's rank correlation). The results of this analysis support the previously mentioned results.

In this sense, the students who considered useful the framework also appreciated the usefulness of the proposed specification language $(r=0.58, p=0.05)$ (see Table 8). In addition, the students who more positively assessed the usefulness of the language were those that better rated the understandability of WA area $(r=0.56, p=0.05)$ and worst the IA area $(r$ $=-0.58, p=0.01)$. As mentioned above, WA is considered as the most understandable area, being IA the least.

In relation to the quality of the solutions to the exercises proposed to the students (see Table 10), it was detected that those who obtained better grades when 
identifying events and devices were those that considered the descriptions proposed as less complex ( $\mathrm{r}$ $=-0.64, p=0.05$ and $r=-0.68, p=0.05$, respectively). On the other hand, students who positively valued the usefulness of the proposed specification language obtained better grades when specifying events $(\mathrm{r}=0.64, \mathrm{p}=0.05)$, actions $(\mathrm{r}=0.63, \mathrm{p}=0.05)$ and devices $(\mathrm{r}=0.58, \mathrm{p}=0.05)$, elements that were among the best identified by the students in the exercises.

\subsection{Discussion}

Gathering all the results above, we can now discuss the testing of the research questions outlined. With regards to Validity (RQ1), the examples of Section 5.2 (CS1, CS2 and CS3) talk about the expressive power of the proposal. If we also consider problems CS4, CS5 and CS6 (section 5.3), the whole set of problems supposes a significant variety of awareness scopes, domains and settings, modalities of interaction, and awareness widgets, as well as devices involved. Particularly, problems CS1, CS5 and CS6 mix explicit interaction with implicit interaction, and problems CS2, CS3, CS4 and CS6 deal with Context and environment awareness, which is less frequent than Workspace awareness. So that we can state that the framework is able to deal with a wide range of awareness support. Results obtained in the practical study confirm this. For example, the engineersstudents judged positively the correctness of the awareness descriptions ( $\mathrm{M}=4.08)$. The research question is also answered by the evaluation of the students' solutions (see Section 5.3.2), which scores in average above the intermediate value of 2.5 $(M=2.97)$ despite of the context of difficulty already discussed.

Concerning the second research question (Ease of use, RQ2), a first positive result is the average complexity $(M=2.23)$ expressed by the students in the questionnaires, which is not too high. However, this result refers to the specific solutions of problems CS1, CS2 and CS3. In more general terms, the students' opinion about the understandability of the framework by awareness areas has been not so good, specifically regarding informal and social awareness $(\mathrm{M}=2.56$ and $\mathrm{M}=2.56$, respectively). The inverse correlation between the usefulness of the framework and the informal awareness understandbility supports this. In this case, we think that the little time available in the preparation of the experience and training activity has influenced these results.
In informal conversations about the quality of the participants' solutions, the expert expressed that he was surprised when reviewing the awareness cards since most of the participants used them with much familiarity despite the complexity of the exercises.

With respect to the fruitfulness of the framework (RQ3), in the case of problems CS1, CS2 and CS3, users found very useful the examples of use, being the catalogue of widgets and devices the most useful component (see Table 8). Interviewed informally about the awareness cards when ending the experience, the students said that they liked them and wondered whether this technique would be used in the subject's lessons next year.

This way, we consider that the research questions RQ1, RQ2 and RQ3 are answered in an affirmative way.

\section{Conclusions}

In this paper, we have developed a framework for the specification of awareness-related information to be used in the analysis phase of the development of systems in which collaborative activities are carried out with both implicit and explicit interactions. By applying this framework, a situation of group work can be characterized. This way, the framework helps to design a system that is intended to deal with one or more awareness dimensions within the ones taken into account. The framework has been defined in terms of a (i) conceptual framework, (ii) a technique for the descriptive specification of awareness-related information and (iii) a process of application.

In order to validate the framework, it has been applied to some cases in which group work is carried out in situations where both implicit and explicit interactions take place. Besides, we have carried out a practical validation with students acting as software engineers. In this study, students have applied the framework to some other cases of collaborative and multimodal work, and they have filled out some questionnaires in order to collect their opinion about the framework. The results of the validation suggest that the framework is a valid tool for objectives outlined, is relatively easy to use, and very useful from the point of view of the users.

As future work, we will use the feedback collected in the practical study in order to improve the framework, for instance in regards to the notation of the awareness cards. Also, we will include new aspects we have not dealt with in the first version of the 
framework and that are quite relevant in awareness, such as emotional aspects, cognitive overload, interruptions and privacy policies. We will as well try to make a better connection between the phases in the process of application of the framework, which would include advancing in the formalization of the descriptive language.

Lastly, the correlation study reveals that engineers that use correctly the events and devices sections of the awareness cards find useful the language and of low complexity the framework. In our opinion, using correlation studies will contribute to understand the behavior of engineers and the use of the technique, and so doing to generate improvements in the approach, for which the study in Section 5.3.3 means a successful analysis scheme.

\section{References}

[1] Antunes P, Herskovic V, Ochoa SF, Pino JA (2014) Reviewing the quality of awareness support in collaborative applications. Journal of Systems and Software 89: 146-169

[2] Bravo C, Duque R, Gallardo J (2013) A groupware system to support collaborative programming: Design and experiences. Journal of Systems and Software 86 (7): 1759-1771

[3] Chen H, Finin T, Joshi A (2003) An Ontology for ContextAware Pervasive Computing Environments. Knowledge Engineering Review, Special Issue on Ontologies for Distributed Sytems.

[4] Collazos C, Guerrero L, Pino J (2003) Knowledge construction awareness. Journal of Student Centered Learning 1(2):76-86

[5] Dey AK (2001) Understanding and Using Context. Personal and Ubiquitous Computing, vol. 5 (1), pp. 4-7.

[6] Dourish P, Bellotti V (1992) Awareness and Coordination in Shared Workspaces. Proc. 1992 ACM Conference on Computer-Supported Cooperative Work.

[7] Ellis C, Gibbs S, Rein G (1991) Groupware: Some Issues and Experiences. Communications of ACM, vol. 34(1). pp. 39-58.

[8] Gallardo J, Molina AI, Bravo C (2012) A framework for the design of awareness support in collaborative situations of implicit interaction. 13th International Conference Interacción Persona-Ordenador, INTERACCION'12. ACM International Conference Proceeding Series.

[9] Gallardo J, Molina AI, Bravo C, Gallego F (2015) Experiences of Use of a Multi-domain Tool for Collaborative Software Engineering Tasks. In J. Filipe and L. Maciaszek (Eds.): Evaluation of Novel Approaches to Software Engineering, CCIS 551, pp. 93-108.

[10] Gallego F, Molina AI, Gallardo J, Bravo C (2011) A conceptual framework for modeling awareness mechanisms in collaborative systems. In: Human-Computer Interaction INTERACT 2011, LNCS 6949, Springer-Verlag.

[11] Greenberg S, Gutwin C (2016) Implications of WeAwareness to the Design of Distributed Groupware Tools. Computer Supported Cooperative Work: CSCW: An International Journal, 25(4-5): 279-293.

[12] Gross T, Stary C, Totter A (2005) User-Centered Awareness in Computer-Supported Cooperative Work-Systems: Struc- tured Embedding of Findings from Social Sciences. International Journal of Human-Computer Interaction 18 (3): 323 360

[13] Gutwin C, Greenberg S, Roseman M (1996) Workspace Awareness in Real-Time Distributed Groupware: Framework, Widgets, and Evaluation. People and Computers XI (Proc. of HCI '96).

[14] Gutwin C, Greenberg S (2002) A Descriptive Framework of Workspace Awareness for Real-Time Groupware. CSCW Journal, vol. 11, pp. 411-446.

[15] Gutwin C, Schneider O, Xiao RB, Brewster S (2011) Chalk Sounds: The Effects of Dynamic Synthesized Audio on Workspace Awareness in Distributed Groupware. Proc. 2011 ACM Conference on Computer Supported Cooperative Work, CSCW 2011.

[16] Hervás R., Bravo J (2011) Towards the ubiquitous visualization: Adaptive user-interfaces based on the Semantic Web. Interacting with Computers 23 (1): 40-56

[17] Jourde F, Laurillau Y, Nigay L (2010) COMM notation for specifying collaborative and multimodal interactive systems Proc. 2nd ACM SIGCHI symposium on Engineering interactive computing systems (EICS '10). ACM, New York, NY, USA, 125-134

[18] Ju W, Lee BA, Klemmer SR (2008) Range: exploring implicit interaction through electronic whiteboard design. Proc. 2008 ACM conference on Computer supported cooperative work (CSCW '08). ACM, New York, NY, USA, 17-26

[19] Lopez G, Guerrero LA (2017) Awareness Supporting Technologies used in Collaborative Systems. Proceedings of the 2017 ACM Conference on Computer Supported Cooperative Work and Social Computing - CSCW '17, pp. 808-820.

[20] López de Ipiña D, Vázquez JI, Abaitua J (2006) A ContextAware Mobile Mash-up for Ubiquitous Web. Proc. 2nd International Workshop UCAmI 2006, pp. 19-34.

[21] Maeda E, Minami Y (2006) Steps toward ambient intelligence. NIT Technical Review, 4 (1).

[22] Mealla S, Väljamäe A, Bosi M, Jordà S (2011) Listening to Your Brain: Implicit Interaction in Collaborative Music Performances. Proc. International Conference on New Interfaces for Musical Expression (NIME 2011): 149-154

[23] Molina AI, Redondo MA, Ortega M (2009) A methodological approach for user interface development of collaborative applications: A case study. Science of Computer Programming, vol. 74 (9), pp. 754-776, 2009.

[24] Molina AI, Gallardo J, Redondo MA, Bravo C (2015) Assessing the awareness mechanisms of a system to support Collaborative Programming. Dyna Colombia 82 (193): 212222.

[25] Molina AI, Gallardo J, Navarro CX, Redondo MA (2016) A Model-Driven Approach for the Development of CSCL Tools that Considers Pedagogical Usability. Proceedings of the 1st International Workshop on Collaborative Modelling in MDE (COMMitMDE 2016), pp. 45-53.

[26] Moody, DL (2009) The "Physics" of Notations: Towards a scientific basis for constructing visual notations in Software Engineering. IEEE Transactions on Software Engineering 35 (6): 756-779.

[27] Obrist M, Velasco C, Vi C, Ranasinghe N, Israr A, Cheok A Spence C, Gopalakrishnakone P (2016) Sensing the future of HCI: touch, taste, and smell user interfaces. Interactions 23 (5): 40-49

[28] Paternò F, Santoro C et al (1998) Formal model for cooperative tasks: Concepts and an application for en-route air traffic control. Proc. 5th Int. Workshop on Design, Specification, and Verification of Intractive Systems DSV-IS '98, Abingdon, Springer-Verlag. 
[29] Paternò F (2004) ConcurTaskTrees: An Engineered Notation for Task Models. The Handbook Of Task Analysis For HCI, D. Diaper and N.A. Stanton (eds.), LEA, Mahwah, NJ. pp. 483-501.

[30] Reinhardt W (2010). A widget-based dashboard approach for awareness and reflection in online learning communities based on Artefact-Actor-Networks. The PLE Conference. Retrieved from http://pleconference.citilab.eu.

[31] Rittenbruch M (2002) Atmosphere: a framework for contextual awareness. International Journal of Human-Computer Interaction 14 (2): 159-180

[32] Schmidt A (2000) Implicit Computer-Human Interaction Through Context. Personal and Ubiquitous Computing, vol. 4, numbers 2-3, pp. 191-199.

[33] Shadbolt N (2003) Ambient Intelligence. IEEE Intelligent Systems, vol. 18, issue 4, pp. 2-3.

[34] Tenenberg J, Roth WM, Socha D (2016) From I-awareness to we-awareness in CSCW. Computer Supported Cooperative Work (CSCW), vol. 25, no. 4-5.

[35] Teruel MA, Navarro E, López-Jaquero V, Montero F, González P (2017) A comprehensive framework for modeling requirements of CSCW systems. Journal of Software: Evolution and Process, 29(5), e1858.

[36] Vázquez JI, López de Ipiña D (2006) SoaM: A Webpowered Architecture for Designing and Deploying Pervasive Semantic Devices. International Journal of Web Information Systems, Vol. 2 (3-4). 